\title{
MAS based on a Fast and Robust FCM Algorithm for MR Brain Image Segmentation
}

\author{
Hanane Barrah \\ Laboratory of Innovative \\ Technologies \\ National School of Applied Sciences \\ Tangier, Morocco
}

\author{
Abdeljabbar Cherkaoui \\ Laboratory of Innovative \\ Technologies \\ National School of Applied Sciences \\ Tangier, Morocco
}

\author{
Driss Sarsri \\ Laboratory of Innovative \\ Technologies \\ National School of Applied Sciences \\ Tangier, Morocco
}

\begin{abstract}
In the aim of providing sophisticated applications and getting benefits from the advantageous properties of agents, designing agent-based and multi-agent systems has become an important issue that received further consideration from many application domains. Towards the same goal, this work gathered three different research fields; image segmentation, fuzzy clustering and multi-agent systems (MAS); and furnished a MAS for MR brain image segmentation that is based on a fast and robust FCM (FRFCM) algorithm. The proposed MAS was tested, as well as the sequential version of the FRFCM algorithm and the standard FCM, on simulated and real normal brains. The experimental results were valuable in both segmentation accuracy and running times point of views.
\end{abstract}

Keywords-agents; MAS; FCM; c-means algorithm; MRI images; image segmentation

\section{INTRODUCTION}

Agent technology has received a significant consideration from many application areas such as computer science, industry and medicine. Thus, developing theories and methods of designing agent-based and multi-agent systems has been adopted by several researchers. in fact, a variety of work has been published in this context. An overview of research and a historical context to the field were presented by N. R. Jennings et al. [1]. The authors concentrated on the interactions (cooperation, coordination and negotiation) within a MAS. Furthermore, they listed the first wave of agent based applications (industrial, commercial, entertainment and medical applications).

In their publication [2], M. Wooldridge and N. R. Jennings pointed out the three main elements needed to design and implement intelligent agents (theories, architectures and languages). They also examined some of the potential applications developed before 1995 (Air traffic control, patient care, believable agents ...).

In 1997, S. Franklin and A. Graesser [3] proposed an agent definition based on the autonomy concept to distinguish software agents from computer programs. Thus, they consider that all software agents are programs and the opposite is not true. The authors fostered their work by discussing two important points. The first one was about the agents classification, where they gave a natural classification of autonomous agents. The second point was an explanation of subagents and societies of agents.
In their review of industrial deployment of MAS, M. Pechoucek and V. Marik [4] presented a detailed list of potential applications of MASs associated to logistics, manufacturing control, production planning, space exploration and other application domains. In spite of the diversity of the applications depicted, the authors concluded that there is a gap between fundamental researchers and industrial users of agent technology.

F. Stonedahl et al. implemented the framework MAgICS as a coherent introductory computer science curriculum based on agent-based model (ABM) and MASs [5]. This framework is composed of nine models spanning seven computer science topics. The authors consider their framework as a starting point for future researches about reinventing introductory computer science education focused on MASs.

In the aim of providing agents able to bilaterally negotiate joint plans with humans, A. Fabregues and C. Sierra [6] proposed a modular software architecture based on an innovative search\&negotiation method and which includes the BDI model (belief, desire and intention). This architecture is able to be extended by incorporating new components, which helps to build skillful agents.

As has been reported previously in literature. The agents and MASs' technology has had a wide range of application domains, this is owing to the beneficial properties of agents such as autonomy, social ability and reactivity [7]. Thus, this work used this recent technology as a solution to the MRI (Magnetic Resonance Imaging) image segmentation problem, which also has been in the center of interest of many researchers for many years. In fact, a wealth of methods have been developed to segment the MRI images [8]. To extract brain tumors, Eman Abdel-Maksoud et al. [9] integrated the kmeans algorithm with its fuzzy version c-means [10], [11]; in order to get benefits from their advantages; and used the median filter as a pre and post-processing to remove noise. As a pre-processing method, the median filter presents two main problems. The first one is increasing the computational time, while the second one lies on the loss of some fine details [12] and which alters the clustering quality in a negative way. To get over this latter limitation and increase the efficiency of the c-means algorithm in presence of noise, several researchers improved it in many ways. the majority tried to include the filtering step in the clustering process by integrating spatial 
information, while the rest tried to modify the dissimilarity measure [13]-[16].

This paper is mainly concentrated on designing a sophisticated MAS for MR brain image segmentation based on a fast and robust FCM algorithm (FRFCM) that includes the median filter into the clustering process. The main idea here, is to utilize the collective work of agents in order to segment the hole brain slices more accurately in a reasonable time.

The remainder of this paper is organized as follows: In section 2 are presented the key concepts of the agents and MASs' field. The FRFCM algorithm and the overall architecture of the proposed MAS are described in section 3. Section 4 is dedicated for some experimental results. A conclusion is presented in section 5 .

\section{Multi-AgEnT Systems : MAS}

Given the degree of interest and the level of activity of the field of agents and MASs, in this section are presented the key concepts needed to design a convenient architecture for MR brain image segmentation.

\section{A. Agents and Multi-agent Systems}

According to various publications in the field of agents and MASs, there is no unique definition of an agent. Although, this lack of definition has not been a bottleneck in the development of this field. In this work, the definition of $\mathrm{M}$. Wooldridge and N. R. Jennings [7] is adopted. Based on this definition, a MAS can be defined as a network of autonomous agents that interact with each other and with their environment to achieve a common goal.

The autonomy is a key concept that has been mentioned, explicitly or implicitly, by several definitions. It is the property that distinguishes an agent from an ordinary program.

\section{B. Agent Architectures}

Architecture is the organization of different elements within a system (agents and environment) and their relationships. Agent architectures is one of the key issues in this field. Indeed, the architecture design has become an interesting research subject of several researchers from different application fields. In literature, many agent architectures have been proposed, they can be roughly categorized into three types: Reactive, deliberative and hybrid architectures.

- Deliberative architectures. Are called also intelligent architectures. They contain a basic knowledge about the environment and can make decisions using a logical reasoning. The best-known deliberative architecture is the BDI architecture [17].

- Reactive architectures. In contrast to deliberative architectures, the reactive ones don't have any basic knowledge of the environment and they don't use any complex symbolic reasoning. In such architectures, the intelligent is seen as the result of interactions between the environment elements. The well-known reactive architecture is subsumption architecture, it was developed by Rodney Brooks in 1985 [18].
- Hybrid architectures. in order to get the best benefits of both deliberative and reactive architectures and to design more complex and sophisticated architectures, the hybrid architectures are more suitable. In fact, they try to combine the best aspects of the above architectures [1]. These architectures are also called layered architectures, because a such architecture may contain two (or more) layers: a deliberative one and a reactive one. The Touring Machines hybrid agent architecture is one of the best-known examples of these architectures, it was developed by Ferguson in 1992 [19].

\section{Interactions within a MAS}

In order to meet the purposes for which a MAS is designed, the agents must be able to interact with each other and with their environment. This interaction can be defined as an exchange of information between agents or between the environment and its agents. Generally, there are three main types of interactions: cooperation, coordination and negotiation.

- Cooperation. Corresponds to a collective work in order to achieve a common goal.

- Coordination. Aims to keep the coherence in the system, thus it seeks to organize the agent activities.

- Negotiation. Seeks to find an agreement that satisfied all the involved agents.

\section{MAS BASED ON A FAST AND ROBUST FCM ALGORITHM}

\section{A. Fast and Robust FCM Algorithm: FRFCM}

In the preceding section, an overview of the key concepts needed to design a convenient MAS is presented. In this section, we turn our attention to a different research area that is not less important than the agents and MASs' research field. It is the clustering problem. Indeed, the clustering is an essential step in several application domains such as data mining and image segmentation. It consists of grouping data into the most homogeneous groups as much as possible [20], [21]. In literature, several methods and techniques about the clustering problem have been developed [20], [21]. However, this work is mainly interested in the fuzzy clustering methods. The best-known fuzzy clustering algorithm is c-means [22], it creates fuzzy clusters by minimizing iteratively an objective function. The major drawback of this algorithm lies on the lack of any spatial information or constraints, which makes it sensitive to noise. In order to overcome this problem and faster the segmentation process, we proposed ; in an earlier work [23]; a fast and robust FCM (FRFCM) algorithm that is a combination of two powerful extensions of the standard FCM algorithm [24], [25].

The FRFCM algorithm takes as input the dataset $\mathrm{D}=\left\{\mathrm{x}_{\mathrm{j}} \in \mathbb{R}\right\}_{\mathrm{j}=1, \ldots, \mathrm{N}}$, the number of clusters $\mathrm{C}$, and minimizes iteratively the following objective function: 


$$
\mathrm{J}(\mathrm{D}, \mathrm{U}, \mathrm{C})=\sum_{\mathrm{i}=1}^{\mathrm{C}} \sum_{\mathrm{j}=1}^{\mathrm{N}} \mathrm{u}_{\mathrm{ij}}^{\mathrm{m}} \cdot\left\|x_{j}-c_{i}\right\|^{2}+\alpha \sum_{\mathrm{i}=1}^{\mathrm{C}} \sum_{\mathrm{j}=1}^{\mathrm{N}} \mathrm{u}_{\mathrm{ij}}^{\mathrm{m}} \cdot\left\|\bar{x}_{j}-c_{i}\right\|^{2}
$$

$\|$.$\| is the Euclidean distance, \mathrm{m}$ is the fuzziness exponent and $\mathrm{U}=\left[\mathrm{u}_{\mathrm{ij}}\right]$ is the fuzzy partition matrix that satisfies the following condition:

$$
\left\{\mathrm{u}_{\mathrm{ij}} \in[0,1] \mid \sum_{\mathrm{i}=1}^{\mathrm{c}} \mathrm{u}_{\mathrm{ij}}=1, \forall \mathrm{j} \text { and } 0<\sum_{\mathrm{j}=1}^{\mathrm{N}} \mathrm{u}_{\mathrm{ij}}<\mathrm{N}, \forall \mathrm{i}\right\} .
$$

$\bar{x}_{j}$ is the median value of the neighbors within a specified window around $\bar{x}_{j}$. The parameter $\alpha$ controls the tradeoff between noise elimination and detail preserving. The minimization of the objective function presented in Eq. 1 is carried out by updating iteratively the fuzzy partition matrix and the cluster centers as follows:

$$
\begin{gathered}
\mathrm{u}_{\mathrm{ij}}=\frac{\left(\left\|\mathrm{x}_{\mathrm{j}}-\mathrm{c}_{\mathrm{i}}\right\|^{2}+\alpha\left\|\overline{\mathrm{x}}_{\mathrm{j}}-\mathrm{c}_{\mathrm{i}}\right\|^{2}\right)^{-\frac{1}{(\mathrm{~m}-1)}}}{\sum_{k=1}^{C}\left(\left\|\mathrm{x}_{\mathrm{j}}-\mathrm{c}_{\mathrm{k}}\right\|^{2}+\alpha\left\|\overline{\mathrm{x}}_{\mathrm{j}}-\mathrm{c}_{\mathrm{k}}\right\|^{2}\right)^{-\frac{1}{(\mathrm{~m}-1)}}} \\
\mathrm{c}_{\mathrm{i}}=\frac{\sum_{\mathrm{j}=1}^{\mathrm{N}} \mathrm{u}_{\mathrm{ij}}^{\mathrm{m}}\left(\mathrm{x}_{\mathrm{j}}+\alpha \overline{\mathrm{x}}_{\mathrm{j}}\right)}{(1+\alpha) \sum_{\mathrm{j}=1}^{N} \mathrm{u}_{\mathrm{ij}}^{\mathrm{m}}}
\end{gathered}
$$

To speed up the clustering process, this work took advantage from the suppressed version [24] of the standard FCM algorithm. The main idea behind this algorithm latter is prizing the biggest memberships and suppressing the others.

Let $x_{j}$ be a pixel and $u_{b j}$ be its degree of belongingness to the $b^{\text {th }}$ cluster. If $u_{b j}$ is the biggest value of all the clusters, then the membership degrees of $\mathrm{x}_{\mathrm{j}}$ will be modified as follows:

$$
\begin{gathered}
\mathrm{u}_{\mathrm{bj}}^{*}=1-\gamma \sum_{\mathrm{i} \neq \mathrm{b}} \mathrm{u}_{\mathrm{ij}}=1-\gamma+\gamma \mathrm{u}_{\mathrm{bj}} \\
\mathrm{u}_{\mathrm{ij}}^{*}=\gamma \mathrm{u}_{\mathrm{ij}}, \mathrm{i} \neq \mathrm{b}
\end{gathered}
$$

Where $\gamma \in[0,1]$.
When $\gamma$ gets closer to 0 (to 1 respectively), the algorithm becomes more hard (fuzzy respectively). Thus, the parameter $\gamma$ balances between the fastness of the hard clustering and the good quality of the fuzzy clustering, in other words, a better selection of $\gamma$ leads to a better clustering quality in a reasonable amount of time. It is always chosen equal to 0.5.

This latest modification has to be done immediately after updating the fuzzy partition matrix in order to bias the membership values to converge rapidly.

\section{Algorithm steps}

Step 0. Fix the clustering parameters (the converging error $\varepsilon$, the fuzziness exponent $\mathrm{m}$ and the number of clusters $\mathrm{C}$ ) and initialize the clusters centers and the new parameters $\alpha$ and $\gamma$.

Step 1. Compute the median filtered image.

Step 2. Update the partition matrix using (Eq. 2).

Step 3. Modify the partition matrix using Eq. 4 and Eq. 5.

Step 4. Update the clusters centers using (Eq. 3).

Repeat steps 2-4 until the following criterion is satisfied:

$$
\left\|\mathrm{C}_{\text {new }}-\mathrm{C}_{\text {old }}\right\|<\varepsilon
$$

\section{B. Agent Based FRFCM Algorithm}

To segment a single slice of the MR brain, the FRFCM algorithm is sufficient, where it furnishes good results in a reasonable amount of time, this is owing to the used spatial information and the balance between hard and fuzzy clustering. To segment the hole slices of an MR brain, the FRFCM algorithm performs slowly because of the big size of data. To get over this problem and provide a sophisticated segmentation system, a multi-agent system based on the agents cooperative work to achieve the global segmentation of the brain is proposed.

It is known that the horizontal brain slices are roughly symmetrical in shape as well as in matter, where if a horizontal slice of a normal brain is split into four equal parts, each part contains necessarily the three brain tissues: white matter, gray matter and cerebrospinal fluid. This fruitful information is the origin of our idea. In fact, we proposed splitting each slice into four equal parts and segment them separately and in a parallel fashion, which can drastically reduce the processing time.

The proposed multi-agent system (Figure 1) takes as input a series of MRI images and return through its output the correspondent segmented images. It is composed of five agents; one master agent that takes control of the system and four slave agents that perform partial segmentations; sharing a memory space and communicating via exchanging ACL (Agent Communication Language) messages; in order to achieve the global segmentation.

The master agent is the first agent created in the platform. When it is created, it gets the data (brain slices) and makes it ready to be used in the shared memory, initializes the 
clustering parameters and creates the slave agents. When all the clustering conditions are verified, the master agent notifies the slave agents in order to start their tasks and waits for them to reply.

Since a slave agent received the master's notification message, it performs the clustering process; using the FRFCM algorithm presented in the previous subsection. When it finishes segmenting its specific part of a given slice, it proceeds automatically to the next slice without waiting for the other agents to finish and when it is done; there is no more slices to process; it notifies the master agent that its mission is accomplished successfully.

When the master agent receives all the slave agents replies, it gathers their partial results in order to form the global one.

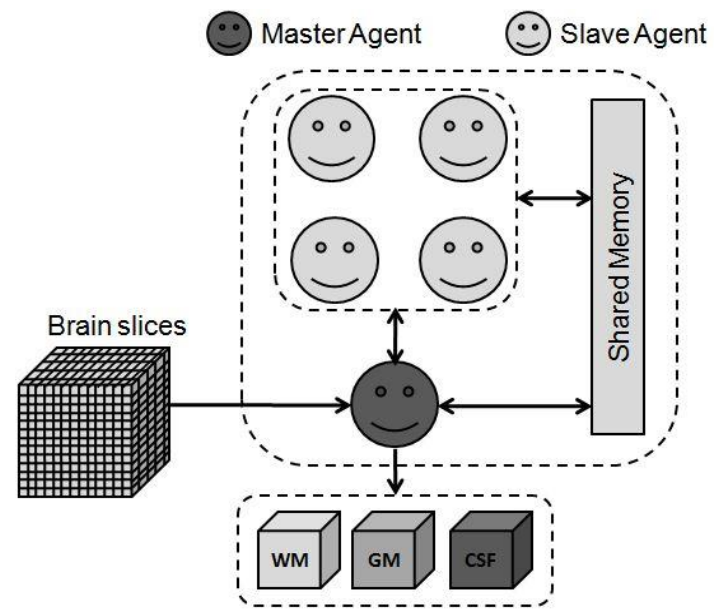

Fig. 1. MAS architecture

For simplification, the proposed MAS is going to be noted in the rest of this paper as FRFCM_MAS.

\section{EXPERIMENTAL RESULTS}

In this section, the proposed MAS is compared with the sequential version of the FRFCM algorithm and the standard FCM in the segmentation accuracy and running times standpoints. In fact, the three approaches were tested on simulated [27] and real [28] normal brains. All the experiments were performed on an Intel Core i7 $(4.4 \mathrm{GHz})$ machine. The proposed MAS was implemented on the JADE middleware [26]. And the clustering parameters were fixed as follows: $\mathrm{C}=3, \varepsilon=10^{-8}, \mathrm{~m}=2, \alpha=4$ and $\gamma=0.5$.

The parameter $\alpha$ is determined by experience; by seeking the interval $\left[\begin{array}{ll}1 & 10\end{array}\right]$; for $\alpha \geq 4$ the changes of the segmentation results are negligible.

As FRFCM and FRFCM_MAS minimize the same objective function, their segmentation results are necessarily very close. To verify this conclusion and show the efficiency of the FRFCM_MAS system over the standard FCM algorithm, we run the three methods on the $90^{\text {th }}$ horizontal slice of a normal brain [27] simulated with $3 \%$ of noise. The segmentation results are presented in Figure 2 and Table 1.

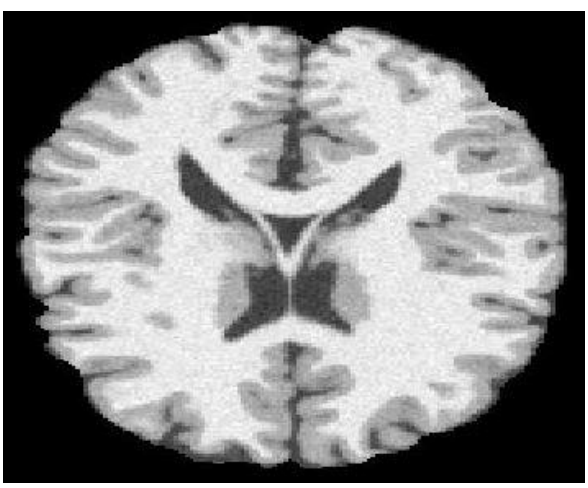

(a)

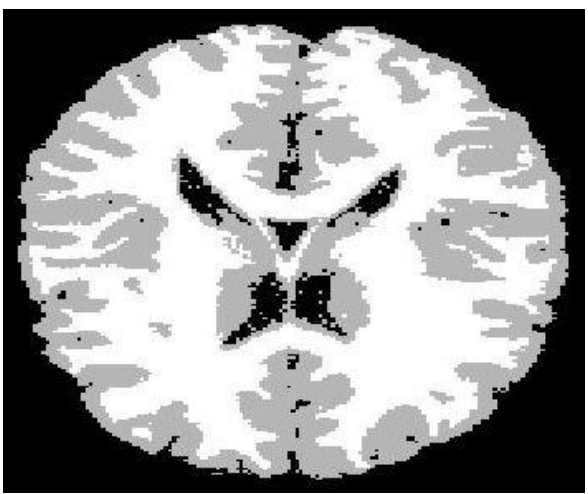

(b)

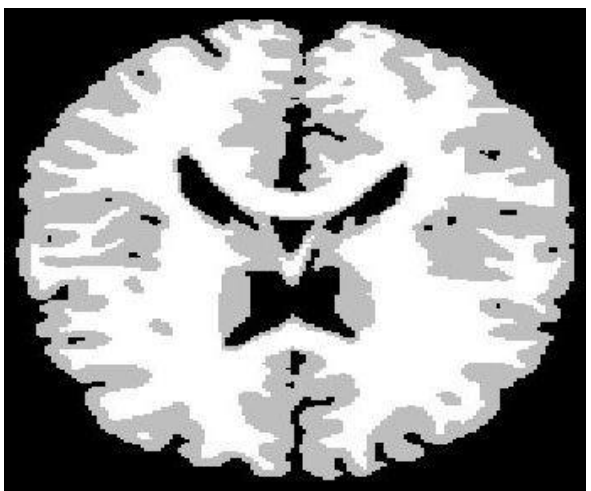

(c)

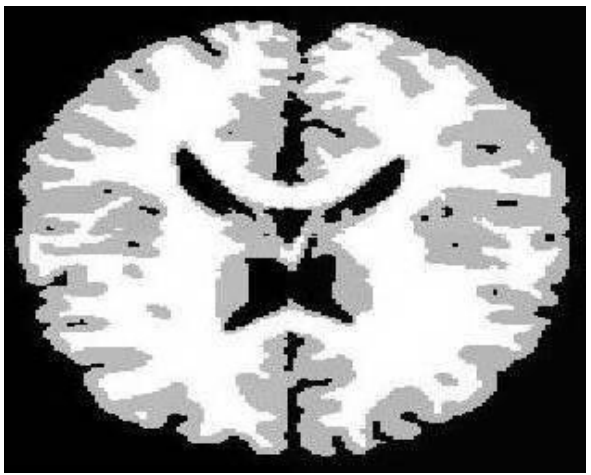

(d)

Fig. 2. Segmentation results. (a) Original image. (b) FCM result. (c) FRFCM result. (d) FRFCM_MAS result 
TABLE I. Clustering Results ON THE $90^{\mathrm{TH}}$ SLICE

\begin{tabular}{|l|c|c|}
\hline \multicolumn{1}{|c|}{ Method } & Misclassification Errors (\%) & $\begin{array}{c}\text { Running } \\
\text { Times (s) }\end{array}$ \\
\hline FCM & 22.43 & 0.79 \\
\hline FRFCM & 19.51 & 0.57 \\
\hline FRFCM_MAS & 19.54 & 0.342 \\
\hline
\end{tabular}

From Figure 2, we notice that the FRFCM and the FRFCM_MAS outperformed the standard FCM algorithm; where they succeeded to some extent to handle noise and extract the most homogeneous regions, and their results are very close, which is also confirmed by the numerical results depicted in Table 1. In fact, the misclassification rate generated by the standard FCM is the biggest one, while the difference between those generated by the FRFCM and FRFCM_MAS is very small, this difference is due to the random initialization. Moreover, from Table 1 , it is remarkable that the proposed MAS performed faster. Thus, the FRFCM_MAS combined between the robustness to noise and the fastness.

To show the strength of the proposed MAS against the sequential version of the FRFCM algorithm, seven experiments were performed on a real normal brain [28], in each experiment the number of the input images is increased. The results are summarized in Figure 3.

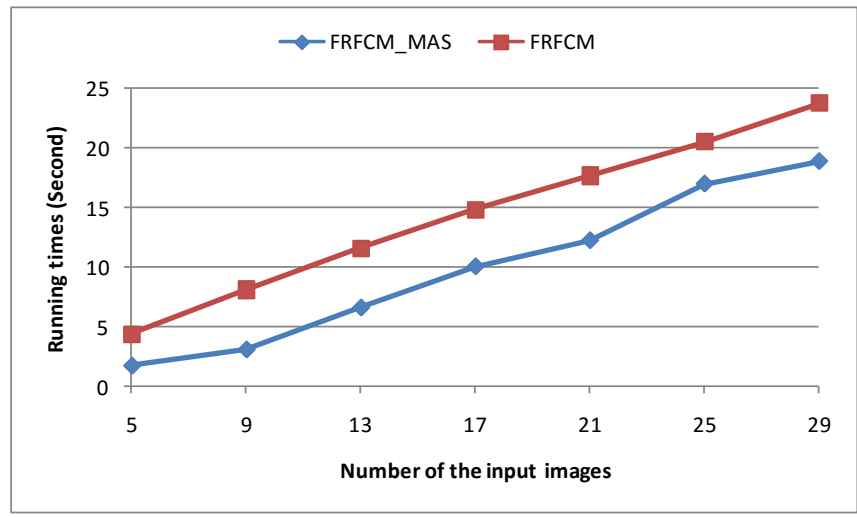

Fig. 3. The running times consumed by both our MAS and the sequential algorithm

From Figure 3, we note that as the number of the input images increases, both methods require much more time. In addition, all the running times performed by the FRFCM_MAS are smaller than those performed by the sequential version of the FRFCM. Which proves the fastness of the proposed method over the sequential version of the FRFCM algorithm.

\section{CONCLUSION}

In this work, the key concepts of the MASs' field have been used along with a fast and robust fuzzy clustering algorithm to design a MAS for segmenting the MR brain images. By testing this MAS as well as the sequential versions of the FRFCM and the FCM on simulated and real normal brains, it showed its robustness and fastness. For this special thanks, the future work will be focused on improving the
FRFCM_MAS system in order to include other image processing techniques such as tumor extraction and volume estimation.

\section{ACKNOWLEDGMENT}

The authors would like to thank the Washington University Alzheimer's Disease Research Center, Dr. Randy Buckner at the Howard Hughes Medical Institute (HHMI) at Harvard University, the Neuroinformatics Research Group (NRG) at Washington University School of Medicine, and the Biomedical Informatics Research Network (BIRN) for making the MRI brain data sets freely available under the following grant numbers: P50 AG05681, P01 AG03991, R01 AG021910, P50 MH071616, U24 RR021382, R01 MH56584.

\section{REFERENCES}

[1] N. R. Jennings, K. Sycara, and M. Wooldridge, “A Roadmap of Agent Research and Development," Auton. Agents Multi-Agent Syst., vol. 1, no. 1, pp. 7-38, Jan. 1998.

[2] M. Wooldridge and N. R. Jennings, "Intelligent agents: Theory and practice,” Knowl. Eng. Rev., vol. 10, no. 02, pp. 115-152, 1995.

[3] S. Franklin and A. Graesser, "Is It an agent, or just a program?: A taxonomy for autonomous agents," in Intelligent Agents III Agent Theories, Architectures, and Languages, J. P. Müller, M. J. Wooldridge, and N. R. Jennings, Eds. Springer Berlin Heidelberg, pp. 21-35, 1997.

[4] M. Pechoucek and V. Marik, "Review of industrial deployment of multiagent systems," Gerstner Lab. Agent Technol. Group Dep. Cybern. Czech Tech. Univ. Prague Czech Repub. Rockwell Autom. Res. Cent. Prague Czech Repub., 2006.

[5] Stonedahl, Forrest, Michelle Wilkerson-Jerde, and Uri Wilensky. "MAgICS: Toward a Multi-Agent Introduction to Computer Science." Multi-Agent Systems for Education and Interactive Entertainment: Design, Use and Experience: Design, Use and Experience, pp. 1-25, 2010 .

[6] A. Fabregues and C. Sierra, "HANA: A Human-Aware Negotiation Architecture,” Decis. Support Syst., vol. 60, pp. 18-28, Apr. 2014.

[7] M. J. Wooldridge, "The logical modelling of computational multi-agent systems," Citeseer, 1992.

[8] D. L. Pham, C. Xu, and J. L. Prince, "Current methods in medical image segmentation 1," Annu. Rev. Biomed. Eng., vol. 2, no. 1, pp. 315-337, 2000.

[9] E. Abdel-Maksoud, M. Elmogy, and R. Al-Awadi, "Brain tumor segmentation based on a hybrid clustering technique," Egypt. Inform. J., vol. 16, no. 1, pp. 71-81, Mar. 2015.

[10] J. C. Bezdek, R. Ehrlich, and W. Full, "FCM: The fuzzy c-means clustering algorithm," Comput. Geosci., vol. 10, no. 2, pp. 191-203, 1984.

[11] M. C. Clark, L. O. Hall, D. B. Goldgof, L. P. Clarke, R. P. Velthuizen, and M. S. Silbiger, "MRI segmentation using fuzzy clustering techniques," Eng. Med. Biol. Mag. IEEE, vol. 13, no. 5, pp. 730-742, 1994.

[12] A. B. Hamza, P. L. Luque-Escamilla, J. Martínez-Aroza, and R. RománRoldán, "Removing noise and preserving details with relaxed median filters," J. Math. Imaging Vis., vol. 11, no. 2, pp. 161-177, 1999.

[13] M. N. Ahmed, S. M. Yamany, N. Mohamed, A. A. Farag, and T. Moriarty, "A modified fuzzy c-means algorithm for bias field estimation and segmentation of MRI data," Med. Imaging IEEE Trans. On, vol. 21, no. 3, pp. 193-199, 2002.

[14] J. Wang, J. Kong, Y. Lu, M. Qi, and B. Zhang, "A modified FCM algorithm for MRI brain image segmentation using both local and nonlocal spatial constraints," Comput. Med. Imaging Graph., vol. 32, no. 8, pp. 685-698, Dec. 2008.

[15] W. Cai, S. Chen, and D. Zhang, "Fast and robust fuzzy c-means clustering algorithms incorporating local information for image segmentation," Pattern Recognit., vol. 40, no. 3, pp. 825-838, 2007. 
[16] Z. Ji, J. Liu, G. Cao, Q. Sun, and Q. Chen, "Robust spatially constrained fuzzy c-means algorithm for brain MR image segmentation," Pattern Recognit., vol. 47, no. 7, pp. 2454-2466, Jul. 2014.

[17] A. S. Rao, M. P. Georgeff, and others, "BDI Agents: From Theory to Practice.," in ICMAS, 1995, vol. 95, pp. 312-319.

[18] R. A. Brooks, "How to build complete creatures rather than isolated cognitive simulators,” Archit. Intell., pp. 225-239, 1991.

[19] I. A. Ferguson, "TouringMachines: An architecture for dynamic, rational, mobile agents," University of Cambridge UK, 1992.

[20] D. Lam and D. C. Wunsch, "Chapter 20 - Clustering," in Academic Press Library in Signal Processing, vol. Volume 1, J. A. K. S., Rama Chellappa and Sergios Theodoridis Paulo S.R. Diniz, Ed. Elsevier, pp. 1115-1149, 2014.

[21] L. Rokach and O. Maimon, "Clustering methods," in Data mining and knowledge discovery handbook, Springer, pp. 321-352, 2005.

[22] S. Ghosh and S. K. Dubey, "Comparative Analysis of K-Means and Fuzzy C-Means Algorithms," IJACSA, vol. 4, pp. 35-38, 2013.
[23] A. Cherkaoui and H. Barrah, "Fast Robust Fuzzy Clustering Algorithm for Grayscale Image Segmentation," in Xth International Conference: on Integrated Design and Production, Dec 2015.

[24] J.-L. Fan, W.-Z. Zhen, and W.-X. Xie, "Suppressed fuzzy c-means clustering algorithm," Pattern Recognit. Lett., vol. 24, no. 9-10, pp. 1607-1612, Jun. 2003.

[25] S. Chen and D. Zhang, "Robust Image Segmentation Using FCM With Spatial Constraints Based on New Kernel-Induced Distance Measure," IEEE Trans. Syst. Man Cybern. Part B Cybern., vol. 34, no. 4, pp. 19071916, Aug. 2004.

[26] F. L. Bellifemine, G. Caire, D. Greenwood, and Wiley InterScience (Online service), Developing multi-agent systems with JADE. Chichester, England; Hoboken, NJ: John Wiley, 2007.

[27] Brainweb, Simulated Brain Database. http://www.bic.mni.mcgill.ca/brainweb/

[28] The Open Access Series of Imaging Studies (OASIS). http://www.oasis-brains.org 